\title{
ROLE OF TLR2 AND TLR4 ON HUMAN NEUTROPHIL FUNCTIONS AGAINST Paracoccidioides brasiliensis
}

Thesis: M. J. Acorci-Valério submitted this thesis for her Doctorate in Tropical Diseases at the Botucatu Medical School, São Paulo State University, UNESP, Botucatu, São Paulo State, Brazil, 2009.

Advisor: Professor Angela Maria Victoriano de Campos Soares

ABSTRACT: In paracoccidioidomycosis, a systemic mycosis caused by the fungus Paracoccidioides brasiliensis $(\mathrm{Pb})$, studies have focused on the role of neutrophils that are involved in the primary response to the fungus. Neutrophil functions are regulated by pro- and anti-inflammatory cytokines. Molecular mechanisms involved in this process are not fully understood, but there are strong evidences about the involvement of toll-like receptors (TLRs). We aimed at evaluating TLR2 and TLR4 expression on human neutrophils activated by GM-CSF, IL-15, TNF-alpha or IFNgamma and challenged with a virulent strain of $P$. brasiliensis (Pb18). Moreover, we asked if these receptors have a role on fungicidal activity, $\mathrm{H}_{2} \mathrm{O}_{2}$ and IL-6, IL-8, TNFalpha and IL-10 production, by activating and challenging cells. All cytokines increased TLR2 and TLR4 expression. Pb18 also increased TLR2 expression, inducing an additional cytokine effect. On the contrary, it inhibited TLR4 expression. All cytokines increased neutrophil fungicidal activity and $\mathrm{H}_{2} \mathrm{O}_{2}$ production; however, this process was not associated with TLR2 or TLR4. Neutrophil activation by GMCSF and TNF-alpha resulted in a significant increase of IL-8 production, while IL-15 and IFN-alpha have no effect. Pb18 also augmented IL-8 expression, inducing an additional effect to that of cytokines. None of the cytokines activated neutrophils by releasing IL-10. This cytokine was only detected after Pb18 challenge. Interestingly, IL-8 and IL-10 production involved TLR2 and mainly TLR4 modulation. The present results suggest that Pb18 interaction with neutrophils through TLR2 and TLR4 with 
consequent IL-8 and IL-10 production may be considered a pathogenic mechanism in paracoccidioidomycosis.

KEY WORDS: neutrophils, immune response, Paracoccidioides brasiliensis, cytokines.

\section{CORRESPONDENCE TO:}

MICHELE JANEGITZ ACORCI VALÉRIO, Departamento de Microbiologia e Imunologia, Instituto de Biociências, UNESP, Botucatu, SP, Brasil. Phone: +55 14 3811 6058. Email: mjanegitz@hotmail.com. 